\title{
Performance Analysis of Impulse Radio Under Timing Jitter Using M-ary Bipolar Pulse Waveform and Position Modulation
}

\author{
Shih-Chun Lin and Tzi-Dar Chiueh \\ Graduate Institute of Electronics Engineering \\ and Department of Electrical Engineering \\ National Taiwan University, Taipei, Taiwan, 10617 \\ E-mail: sclin@analog.ee.ntu.edu.tw, chiueh@cc.ee.ntu.edu.tw.
}

\begin{abstract}
In this paper, we derive analytical bit error rate (BER) performance of impulse radio using spread-spectrum technique under correlated random timing jitters. The result is applied to the bipolar pulse waveform and position modulation (BPWPM) to study its degradation in performance. High-order derivatives of the Gaussian pulse with about $500 \mathrm{ps}$ pulse width are chosen to comply the FCC part 15 limit. The analysis concludes that both jitter root-mean square (RMS) value and correlation between jitters are important factors that influence system performance. When the jitters are highly correlated, different signal sets will have different levels of performance degradation even with the same jitter RMS value, which needs be taken into consideration in impulse radio system design.
\end{abstract}

\section{INTRODUCTION}

By transmitting nano-second duration, low duty-cycle baseband pulses, ultra-wide band (UWB) impulse radio is a prominent candidate for low-cost short-range wireless communication application However, as indicated in [1], impulse radio will induce large interference to global position system (GPS) under the original FCC part 15 level. Thus FCC has further restricted the part 15 mask for communication usage to $3.1-10.6 \mathrm{GHz}$ [2]. Without frequency-translation mixer in the transmitter, the original Gaussian monocycle pulse used in [1], [3] has too wide a bandwidth that violates the revised FCC mask. This motivates us to explore the impact of pulse waveform and consider waveform as a parameter in system design.

The other issue that affects UWB system performance significantly and strongly depends on pulse waveform is pulse timing precision. The impact of independent jitter on impulse radio systems was simulated in [3] and the performance degradation is more serious than that in narrowband systems. In practice, correlated jitter sources such as substrate and supply noise are commonly seen in transmitter/receiver circuits [4], [5]. Thus we take the correlation between jitters into consideration and study the impact of correlated jitters on the BER performance in additive white Gaussian noise (AWGN) channel. The results can be used as a theoretical upper bound for evaluating system performances in multipath channels.

This paper is organized as follows. In Section II we will introduce the impulse radio system and signal model. Next, signal set design is presented in Section III. Section IV provides the analysis of the impact caused by correlated random jitters on error performance with numerical examples given in Section V. Finally, Section VI concludes this paper.

This work was supported in part by MediaTek Inc.

\section{System AND SIGNAL MODEL}

\section{A. Channel Model and Pulse Waveform}

Consider a single-user system and assume that the timedomain characteristic of the antenna is a differentiator. The pulse propagated in the channel is $P_{T}(t)=\int_{-\infty}^{t} P(\zeta) d \zeta$, and the received pulse in the baseband processor is $A P(t-\tau)+$ $n(t)$, where $P(t), A$, and $\tau$ represent the pulse waveform, the link path loss, and the path delay, respectively. The noise process $n(t)$ is an additive white Gaussian process with twosided power spectral density $N_{0} / 2$.

As in [1], we select those pulse waveforms derived from the Gaussian pulse for detailed study. These waveforms are widely used in image and spatial vision processing [6]. The $k$ th derivative of the Gaussian pulse is expressed as

$$
G_{k}(t)=A_{k} \frac{d^{(k)}}{d t^{(k)}} e^{-2 \pi\left(t / t_{n}\right)^{2}}
$$

where the pulse width $T_{p}$ is about $2.5 t_{n}$ and the pulse energy $E_{p}=\int_{-\infty}^{+\infty}[P(\zeta)]^{2} d \zeta$ is normalized to unity through amplitude $A_{k}$. As indicated in [1], increasing the order of the derivative while keeping the pulse width unchanged will shift the power spectral density toward higher frequency and induce less interference below $3.1 \mathrm{GHz}$. To this end, we choose higher-order $G_{6}(t)$ and $G_{7}(t)$ with $t_{n}=210 \mathrm{ps}$ and they both comply the FCC part 15 limit.

\section{B. Spread Spectrum Signal Format}

To combat the narrowband interference from existing systems, conventionally spread-spectrum techniques are used in UWB signaling. In this paper, we adopt the DSSS-UWB signaling format as described in [7]. We first define $S_{i}(t)$ as a possible symbol, and consider the bipolar DSSS-UWB signal, which can be represented as

$$
S_{i}(t)=a_{i} \sum_{n=0}^{N_{s}-1}\left(c_{n}^{D}\right) P_{i}\left(t-n T_{f}-\delta_{i}\right)
$$

where $a_{i}$ is the polarity; $N_{s}$ is the number of pulses per symbol (spreading factor); $c_{n}^{D}$ is the $n$th chip of the spreading sequence; $T_{f}$ is the pulse repetition (frame) time and $\delta_{i}$ is the pulse offset. Note that each chip in the spread-spectrum signal is waveform-mapped to a single pulse. One feature of impulse radio is that we can let $T_{f} \gg T_{p}$ to obtain the extra processing gain $10 \log _{10}\left(T_{f} / T_{p}\right)$ in addition to the spreading factor. The spreading sequence is a pseudorandom $(\mathrm{PN})$ code to provide resistance against interference. 
If the receiver hardware complexity is the main system consideration, Golay sequence is suggested over conventional PN codes [8].

\section{Signal SET DESIGN}

In this section, we will introduce the signal set design criteria and resultant design. The signal set design parameters corresponding to the data modulation are

$$
\begin{aligned}
& a_{i} \in\{-1,+1\} \\
& P_{i}(t) \in\left\{W_{0}(t), W_{1}(t), \ldots W_{d_{w}-1}(t)\right\} \\
& \delta_{i} \in\left\{\tau_{0}=0<\tau_{1}<\cdots<\tau_{d_{s}-1}\right\}
\end{aligned}
$$

where $d_{w} \geq 1$ and $d_{s} \geq 1$ are integers that represent the dimension of waveforms $P_{i}(t)$ and timing offset $\delta_{i}$, respectively. The size of the signal set is given by $M=2 \cdot d_{w} \cdot d_{s}$.

The error performance of the signal set depends on the normalized correlation value between symbols $S_{i}(t)$ and $S_{j}(t)$. Assuming that the receiver is synchronized and the spreading sequence $c_{n}^{D}$ is known, the correlation between two symbols is

$$
\begin{aligned}
\rho_{i j} & =\frac{1}{E_{s}} \int_{-\infty}^{+\infty} S_{i}(\zeta) S_{j}(\zeta) d \zeta \\
& =a_{i} a_{j} \cdot R_{i j}\left(\delta_{j}-\delta_{i}\right),
\end{aligned}
$$

where the normalized pulse cross-correlation function $R_{i j}(\tau)$ is

$$
R_{i j}(\tau)=\frac{\int_{-\infty}^{+\infty} P_{i}(t) P_{j}(t-\tau) d t}{\sqrt{E_{p_{i}} E_{p_{j}}}} .
$$

With the defined correlation value, we then study the signal set performance using union bound for the symbol error probability (SER) [9]. With perfectly synchronized spread spectrum signals in the optimal coherent detector under freespace channel and equally likely signals assumption, the union bound gives

$$
\begin{aligned}
U B P_{e} & =\frac{1}{M} \sum_{i=0}^{M-1} \sum_{j=0, j \neq i}^{M-1} P_{e}\left(S_{i} \rightarrow S_{j}\right) \\
& =\frac{1}{M} \sum_{i=0}^{M-1} \sum_{j=0, j \neq i}^{M-1} Q\left(\sqrt{\left(1-\rho_{i j}\right) \cdot b \gamma_{b}}\right),
\end{aligned}
$$

where $P_{e}\left(S_{i} \rightarrow S_{j}\right)$ is the pairwise symbol error probability of transmitting $S_{i}(t)$ and deciding erroneously to $S_{j}(t) ; Q(\cdot)$ is the Gaussian tail integral; $b=\log _{2} M$ and the received signal to noise ratio (SNR) per bit $\gamma_{b}$ is $A^{2} E_{s} /\left(N_{0} b\right)$.

When $M=2$, it is well known that antipodal signal set achieves minimum symbol error rate performance. It can be shown that for the case $M>2$ with parameters in (3), the minimum $U B P_{e}$ occurs when the signal set is biorthogonal. From (4), this can be done by choosing disjoint time slots, i.e. letting $\delta_{j}-\delta_{i}$ be sufficiently larger than $T_{p}$. Here we define a design parameter $T_{O R}$ as the minimum $\delta_{j}-\delta_{i}$ that satisfies this condition. When two overlapping pulse waveforms, $P_{i}(t) \in\left\{W_{0}(t), W_{1}(t)\right\}$, are used, we can also make $\rho_{i j}=0$ by letting $\delta_{j}-\delta_{i}=0$ and $\int_{-\infty}^{-\infty} W_{0}(t) W_{1}(t) d t=$ 0 . For example, if $W_{0}(t)=G_{6}(t)$ and $W_{1}(t)=G_{7}(t)$, this condition can be satisfied.
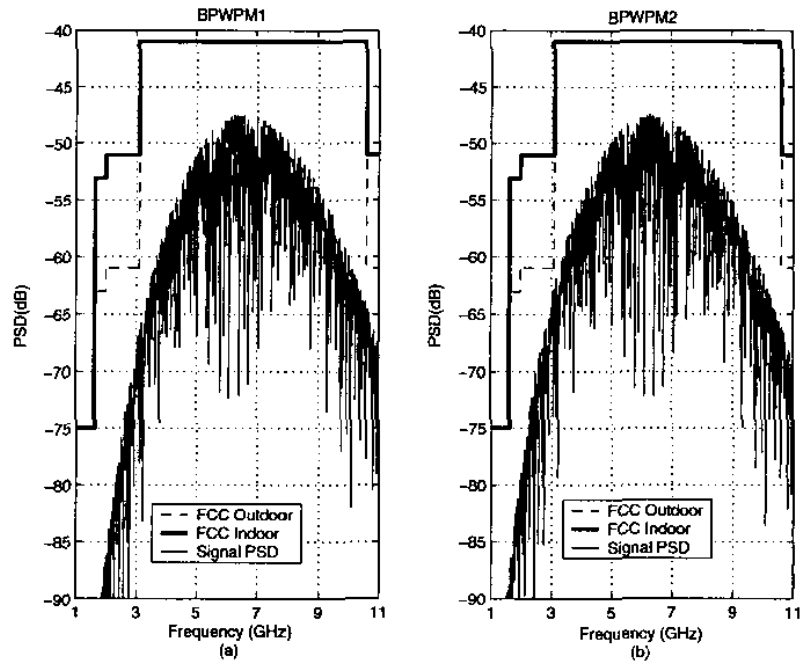

Fig. 1. Power spectral densities of the (a) BPWPM1 and (b) BPWPM2 signals using the Golay sequence with code length 256.

The resultant signal sets are summarized in Table I. Fig. 1 shows the transmitted signal power spectral densities of these signals. As the design in [7] that intends to ease the sensitivity loss in other narrowband systems from impulse radio signals, we set signal PSD to be $6 \mathrm{~dB}$ lower than the FCC part 15 mask. Since the BER performance is more relevant in digital communication, we use the union bound on BER given by

$$
U B P_{b}=\frac{1}{M} \sum_{i=0}^{M-1} \sum_{j=0, j \neq i}^{M-1} P_{e}\left(S_{i} \rightarrow S_{j}\right) \cdot \frac{d_{H}(i, j)}{b},
$$

where $d_{H}(i, j)$ is the Hamming distance between bit patterns assigned to $S_{i}(t)$ and $S_{j}(t)$.

\section{Performance Analysis Under Correlated RANDOM TIMING JITTERS}

In this section, we will derive formulas that describe the impact of correlated random timing jitters on the BER performance of different modulation schemes listed in Table I. To simplify the theoretical analysis and notation, the following additional assumptions are made:

1) The receiver is synchronized and there is no inter-chip interference (ICI) caused by small timing jitter. The spreading sequence $c_{n}^{D}$ will then have no effects in the analysis, so we set $c_{n}^{D}=1, \forall n$ for simplicity.

2) The sequence of random timing jitters $\varepsilon_{0}, \ldots, \varepsilon_{n}, \ldots$ are samples of a zero-mean wide-sense stationary Gaussian process with variance $\sigma_{\varepsilon}^{2}$ for all $\varepsilon_{n}$ [3], [5]. To model the band-limited phenomenon of this process, we assume this process is non-white with autocorrelation function which has essentially zero values when the relative time difference between samples are longer than a certain threshold, i.e. $E\left[\left(\varepsilon_{n}\right)\left(\varepsilon_{l}\right)\right]=0$, when $|l-n| \geq m$. In other words, induced jitters will be correlated over several adjacent pulses, and they are independent for pulses farther apart. Furthermore, 
TABLE I

SUMmary of M-ARy Bipolar PUlse Waveform Position Modulation Family.

\begin{tabular}{|c|c|c|c|c|}
\hline $\begin{array}{l}\text { Type of } \\
\text { signal }\end{array}$ & $\begin{array}{c}\text { Signal pattern } \\
i=0, \ldots, M-1\end{array}$ & $\begin{array}{c}\text { Size of } \\
\text { signal set } M\end{array}$ & $\begin{array}{l}\text { Union bound } \\
\text { BER } U B P_{b}\end{array}$ & $\begin{array}{l}\text { Correlators } \\
\text { in receiver }\end{array}$ \\
\hline BPWPM1 & $\begin{aligned} P_{i} & =G_{7} \forall i \\
\delta_{i} & =[i \bmod (M / 2)] T_{O R}\end{aligned}$ & $2\left\lfloor\frac{T_{f}}{T_{O R}}\right\rfloor$ & $Q\left(\sqrt{2 \cdot b \gamma_{b}}\right)+\frac{M-2}{2} Q\left(\sqrt{b \gamma_{b}}\right)$ & 1 \\
\hline BPWPM2 & $\begin{aligned} P_{i} & =\left\{\begin{array}{l}G_{6}\left\lfloor\frac{i}{(M / 4)}\right\rfloor \in o d d \\
G_{7} \text { else }\end{array}\right. \\
\delta_{i} & =[i \bmod (M / 4)] T_{O R}\end{aligned}$ & $4\left\lfloor\frac{T_{f}}{T_{O R}}\right\rfloor$ & $Q\left(\sqrt{2 \cdot b \gamma_{b}}\right)+\frac{M-2}{2} Q\left(\sqrt{b \gamma_{b}}\right)$ & 2 \\
\hline
\end{tabular}

* All $a_{i}=-1^{\left\lfloor\frac{i}{(M / 2)}\right\rfloor}$ in both signal sets.

the spreading factor $N_{s}$ is larger than the threshold, $N_{s} \gg m \geq 1$.

The objective now is to compute the general $U B P_{b}$ in (7) in the case of such timing jitters. We first compute the individual pairwise SER, $P_{e}\left\{S_{i} \rightarrow S_{j}\right\}$. The received signal $r(t)$ at the correlator input is

$$
r(t)=A \cdot a_{i} \sum_{n=0}^{N_{s}-1} P_{i}\left(t-\tau-n T_{f}-\delta_{i}\right)+n(t),
$$

and the correlator template waveform is

$$
v(t)=\sum_{n=0}^{N_{s}-1} v_{i j}\left(t-\tau-n T_{f}-\varepsilon_{n}\right),
$$

where

$$
v_{i j}(t)=a_{i} P_{i}\left(t-\delta_{i}\right)-a_{j} P_{j}\left(t-\delta_{j}\right) .
$$

The decision metric $y_{i j}$ at the output of the correlator is

$$
y_{i j}=\int_{t \in T} r(t) v(t) d t=d_{i j}+n_{i j},
$$

where $T$ is the symbol time, and $d_{i j}$ and $n_{i j}$ are the data component and the noise component, respectively. An error is made when the decision metric is less than zero, i.e. $y_{i j}<$ 0 .

The receiver is confronted with an $M$-ary hypothesis testing problem with hypothesis $H_{i}: A \cdot S_{i}(t-\tau)+n(t), 0 \leq$ $i<M-1$. To compute the pairwise SER $P_{e}\left\{S_{i} \rightarrow S_{j}\right\}=$ $P\left(y_{i j}<0 \mid H_{i}\right)$, we need the statistics of both $d_{i j}$ and $n_{i j}$. The noise component $n_{i j}$ is $\int_{t \in T} n(t) v(t) d t$, and it can be shown that this random variable is independent of random jitter $\varepsilon_{n}$ and is normally distributed with distribution

$$
n_{i j} \sim N\left(0, N_{0} N_{s}\left(1-\rho_{i j}\right)\right)
$$

where $Z \sim N\left(\mu, \sigma^{2}\right)$ means that random variable $Z$ is a normal distribution with mean $\mu$ and variance $\sigma^{2}$. The statistics of data component $d_{i j}$ is far more complex, we first write it as

$$
\begin{aligned}
d_{i j} & =A \int_{t \in T} \sum_{n=0}^{N_{s}-1} a_{i} P_{i}\left(t-\tau-n T_{f}-\delta_{i}\right) v_{i j}\left(t-\tau-n T_{f}-\varepsilon_{n}\right) d t \\
& =A \sum_{n=0}^{N_{s}-1}\left\{R_{i}\left(\varepsilon_{n}\right)-a_{i} a_{j} R_{i j}\left(\delta_{j i}+\varepsilon_{n}\right)\right\}=A \sum_{n=0}^{N_{s}-1} f_{i j}\left(\varepsilon_{n}\right)
\end{aligned}
$$

where $R_{i}(\tau)$ is the autocorrelation function of $P_{i}(t)$ obtained from setting $P_{j}(t)=P_{i}(t)$ in (5) and $\delta_{j i}=\delta_{j}-\delta_{i}$ is the relative timing offset between $S_{i}(t)$ and $S_{j}(t)$. Here we define a new function $f_{i j}(\cdot)$ to simplify the notation. Since the waveforms used in impulse radio system are not simple rectangular pulses, $f_{i j}(\tau)$ is usually a nonlinear function in its argument.

From the above derivations, we know that the data component $d_{i j}$ is summation of nonlinear functions of correlated random variables $\varepsilon_{n}$, typically it is hard to identify the statistics of such complex combination of random variables. Fortunately, $f_{i j}(\cdot)$ is a zero-memory nonlinear function, and the sequence of random variables $f_{i j}\left(\varepsilon_{n}\right), 0 \leq n<N_{s}$ has the following properties:

1) Sequence $f_{i j}\left(\varepsilon_{n}\right)$ is stationary since the sequence of jitters $\varepsilon_{n}$ is stationary.

2) It can be shown that sequence $f_{i j}\left(\varepsilon_{n}\right)$ is m-dependent sequence of random variables since sequence $\varepsilon_{n}$ can be modelled as $\mathrm{m}$-dependent. The definition of $\mathrm{m}$ dependent sequence of random variables can be found in $[10$, p. 215].

3) Let $E[$.$] be the expectation function, then E\left[\left|f_{i j}\left(\varepsilon_{n}\right)\right|^{3}\right]$ exists and is bounded in the range from 0 to $2^{3}$ since $\left|f_{i j}\left(\varepsilon_{n}\right)\right| \leq 2$.

Based on these three properties, we can invoke the central limit theorem for m-dependent sequence of random variables in [10] to compute the statistics of $d_{i j}$, and

$$
d_{i j} \sim N\left(A N_{s} \mu_{i j}, A^{2} N_{s} \sigma_{i j}^{2}\right),
$$

where

$$
\begin{aligned}
& \mu_{i j}=E\left[f_{i j}\left(\varepsilon_{0}\right)\right] \\
& \sigma_{i j}^{2}=\operatorname{Var}\left[f_{i j}\left(\varepsilon_{0}\right)\right]+2 \sum_{n=1}^{m} \operatorname{Cov}\left[f_{i j}\left(\varepsilon_{0}\right) f_{i j}\left(\varepsilon_{n}\right)\right],
\end{aligned}
$$

where $\operatorname{Var}[\cdot]$ denotes the variance of a random variable and $\operatorname{Cov}[\cdot]$ the covariance of two random variables.

From the statistics of data component $d_{i j}$ in (10) and noise component $n_{i j}$ in (8) and the fact that they are independent Gaussian random variables, the resulting error probability $P_{e}\left\{S_{i} \rightarrow S_{j}\right\}=P\left(d_{i j}+n_{i j}<0 \mid H_{i}\right)$ is

$$
Q\left(\sqrt{\frac{A^{2} N_{s}^{2} \cdot \mu_{i j}^{2}}{N_{0} N_{s}\left(1-\rho_{i j}\right)+A^{2} N_{s} \sigma_{i j}^{2}}}\right),
$$


where we consider only the case when $\mu_{i j}>0$. Basically, this formula is sufficient to illustrate the pairwise SER behavior since parameters $\mu_{i j}$ and $\sigma_{i j}^{2}$ can be computed using numerical integral with known probability density function (PDF) of $\varepsilon_{0}$ and joint PDF of $\varepsilon_{0}$ and $\varepsilon_{n}$. However, this formula carries less insight and requires long computation time on the double integral. To this end, we use the Taylor series expansion on the nonlinear $f_{i j}(\cdot)$ with moment functions of random jitters to estimate $\mu_{i j}$ and $\sigma_{i j}^{2}$. The estimation of mean in (10) using $K_{m}$-order approximation is

$$
\begin{aligned}
E\left[f_{i j}\left(\varepsilon_{0}\right)\right] & \approx\left(1-\rho_{i j}\right)+\sum_{k=1}^{K_{m}} \frac{f_{i j}^{(k)}(0)}{k !} E\left[\left(\varepsilon_{0}\right)^{k}\right] \\
& =\left(1-\rho_{i j}\right)+L_{i j},
\end{aligned}
$$

where $f_{i j}^{(k)}(\cdot)$ is the $k$ th derivative of $f_{i j}(\cdot)$ and $L_{i j}$ represents the effect of jitter on $\mu_{i j}$. Note that $f_{i j}^{(0)}(0)=1-\rho_{i j}$. Covariance can also be estimated with $K_{\nu}$ th Taylor polynomial in two variables

$$
\begin{aligned}
& \operatorname{Cov}\left[f_{i j}\left(\varepsilon_{0}\right) f_{i j}\left(\varepsilon_{n}\right)\right] \\
& \approx \sum_{k=1}^{K_{v}} \sum_{q=1}^{K_{v}-k} \frac{f_{i j}^{(k)}(0)}{k !} \frac{f_{i j}^{(q)}(0)}{q !}\left(E\left[\left(\varepsilon_{0}\right)^{k}\left(\varepsilon_{n}\right)^{q}\right]-E\left[\left(\varepsilon_{0}\right)^{k}\right] E\left[\left(\varepsilon_{n}\right)^{q}\right]\right) .
\end{aligned}
$$

Now the problem becomes finding the joint moment of random values $\varepsilon_{0}$ and $\varepsilon_{n}, E\left[\left(\varepsilon_{0}\right)^{k}\left(\varepsilon_{n}\right)^{q}\right]$. Since they are jointly Gaussian this joint moment can be found in [11, p. 212], which is a polynomial function of $\sigma_{\varepsilon}$ and $\rho_{\varepsilon, n}=$ $E\left[\left(\varepsilon_{0}\right)\left(\varepsilon_{n}\right)\right] / \sigma_{\varepsilon}^{2}$. Moment function of $\varepsilon_{0}, E\left[\varepsilon_{0}^{k}\right]$ in (12) is a special case of $E\left[\left(\varepsilon_{0}\right)^{k}\left(\varepsilon_{n}\right)^{q}\right]$, which can be obtained by setting $\varepsilon_{n}=\varepsilon_{0}$ and $q=0$. Replacing (12) and (13) into (10), we conclude that the effect of Gaussian random jitter sequence on $\mu_{i j}$ and $\sigma_{i j}^{2}$ can be approximated by polynomial functions of jitter variance $\sigma_{\varepsilon}^{2}$ and correlation $\rho_{\varepsilon, n}$. The influences of pulse waveform are reflected on the polynomial coefficients through nonlinearity $f_{i j}(\cdot)$.

Finally, the pairwise SER is given by

$$
Q\left(\sqrt{\frac{\left[\left(1-\rho_{i j}\right)+L_{i j}\right]^{2}}{\left(1-\rho_{i j}\right)+\frac{b \gamma_{b}}{N_{s}} V_{i j}} \cdot b \gamma_{b}}\right)=Q\left(\sqrt{S N R_{i j}^{d}}\right)
$$

where $V_{i j}$ denotes the approximation of $\sigma_{i j}^{2}$. When no jitter exists, $\sigma_{\varepsilon}=0$ then $L_{i j}$ and $V_{i j}$ will be equal to zero, thus the pairwise SER reduces to the case in (6). As shown in (14), the effect of random jitters can be treated as another additive Gaussian noise source with non-zero mean and SNR related variance.

The performance for signal sets listed in Table I using (14) will now be discussed. In these modulation schemes, three different cases of pairwise SER are considered. We can observe that in these cases with $P_{i}(t)=G_{k}(t)$ and $P_{j}(t)=G_{q}(t)$, all $f_{i j}(\tau)$ in (9) can be reduced to or expressed in a common form, i.e. $R_{G_{k}}(\tau)-\theta \cdot R_{G_{k} G_{q}}(\tau)$, where $\theta=0$ or $\pm 1, R_{G_{k}}(\tau)$ is Gaussian pulse autocorrelation function and $R_{G_{k} G_{q}}(\tau)$ is Gaussian pulse cross-correlation function, respectively. Thus we can define two new variables, $L_{k q}(\theta)$ and $V_{k q}(\theta)$ to respectively denote $L_{i j}$ and $V_{i j}$ when $f_{i j}(\tau)$ equals to the above form.
The first case is $S_{i}(t)=-S_{j}(t)$, i.e. the two signals are antipodal with $P_{i}(t)=G_{k}(t)$ and $f_{i j}(\tau)$ equals to $2 R_{G_{k}}(\tau)$ ( $k=7$ in BPWPM1 and $k=6,7$ in BPWPM2 signal set). According to (14) with $\rho_{i j}=-1, L_{i j}=2 L_{k k}(0)$ and $V_{i j}=$ $4 V_{k k}(0)$, the resulting $S N R_{i j}^{d}$ for the antipodal signal set is

$$
S N R_{A N}^{k}=\frac{\left[1+L_{k k}(0)\right]^{2}}{\frac{1}{2}+\frac{b \gamma_{b}}{N_{s}} V_{k k}(0)} b \gamma_{b}
$$

Note that $L_{k k}(0)$ is always less than zero since signal autocorrelation functions are always maximum and concave at $\tau=0$, i.e. $S N R_{A N}^{k}$ is always smaller than the perfect timing case. In addition, the autocorrelation functions reach global extremum and $f_{i j}^{(1)}(0)$ is always zero at $\tau=0$, and $V_{k k}(0)$ is related only to the higher power (larger than one) of jitter variance $\sigma_{\varepsilon}^{2}$.

The second case is orthogonal signal pair obtained by choosing disjoint time slots between two signals. Since signal correlation values of Gaussian pulses are almost zero when the relative timing offset is larger than or equal to $T_{O R}$, thus the term $R_{i j}\left(\delta_{j i}+\varepsilon_{n}\right)$ in (9) can be neglected for small jitter $\varepsilon_{n}$. When $P_{i}(t)=G_{k}(t)$, we can set $f_{i j}(\tau)$ to $R_{G_{k}}(\tau)$. Using this fact together with $\rho_{i j}=0$ in (14), the $S N R_{i j}^{d}$ for this kind of orthogonal signal set is

$$
S N R_{D O R}^{k}=\frac{\left[1+L_{k k}(0)\right]^{2}}{1+\frac{b \gamma_{b}}{N_{s}} V_{k k}(0)} b \gamma_{b} .
$$

Note that $S N R_{D O R}^{k}$ is almost the same as $S N R_{A N}^{k}$ except that the noise power is twice as large.

The third case is the overlapped orthogonal signal sets. From $\delta_{j i}=0$ together with $P_{i}(t)=G_{k}(t), P_{j}(t)=G_{q}(t)$, and $a_{i} a_{j}=+1, L_{i j}$ and $V_{i j}$ in (14) are $L_{k q}(+1)$ and $V_{k q}(+1)$, respectively. Then the $S N R$ becomes

$$
S N R_{O O R}^{+k q}=\frac{\left[1+L_{k q}(+1)\right]^{2}}{1+\frac{b \gamma_{b}}{N_{s}} V_{k q}(+1)} b \gamma_{b},
$$

where $k=6, q=7$ or $k=7, q=6$ in BPWPM2 system. When $a_{i} a_{j}=-1$, the SNR is $S N R_{O O R}^{-k q}$, which is the same as $S N R_{O O R}^{+k q}$ except the arguments in $L_{k q}$ and $V_{k q}$ are both -1 . Substituting (15) through (17) in (7), we then summarize the union bound on BER in the presence of jitter for the two signal sets in Table II.

\section{NUMERICAL EXAMPLE}

In this section we compute the BER under 20 ps RMS jitter using Table II. To make a fair comparison, we fix the symbol time $T=N_{s} T_{f}$ and $M=16$ for both signal sets. As can be seen in Table I, BPWPM2 signals can have half $T_{f}$ as BPWPM1 at the same $M$ thus having twice $N_{s}$. So we set $N_{s}=128$ for BPWPM1 and $N_{s}=256$ for BPWPM2 signals. The correlated time window $m$ is affected by $T_{f}$ and the bandwidth of the jitter process, and typically it is on the order of tens. This research covers a selection of $m$ which is small compared to $N_{s}$. Since BPWPM2 signals have half $T_{f}$ as BPWPM1 and thus twice $m$, we set $m=10$ for BPWPM1 and $m=20$ for BPWPM2. The bandlimited effect is modelled as first-order low-pass filtering and $\rho_{\varepsilon, n}$ 
TABLE II

UNION BOUND BER UNDER JITTERS FOR THE TWO SIGNAL SETS

\begin{tabular}{cc}
$\begin{array}{c}\text { Type } \\
\text { of signal }\end{array}$ & $\begin{array}{c}\text { Union bound BER } \\
\text { under random timing jitters }\end{array}$ \\
\hline BPWPM1 & $Q\left(\sqrt{S N R_{A N}^{7}}\right)+\frac{M-2}{2} \cdot Q\left(\sqrt{S N R_{D O R}^{7}}\right)$ \\
\hline BPWPM2 & $\frac{1}{2} \cdot\left[Q\left(\sqrt{S N R_{A N}^{7}}\right)+Q\left(\sqrt{S N R_{A N}^{6}}\right)\right]+$ \\
& $\frac{1}{2 b} \cdot\left[Q\left(\sqrt{S N R_{O O R}^{+76}}\right)+Q\left(\sqrt{S N R_{O O R}^{+67}}\right)\right]+$ \\
& $\frac{M-4}{4} \cdot\left[Q\left(\sqrt{S N R_{O O R}^{-76}}\right)+Q\left(\sqrt{S N R_{O O R}^{-67}}\right)\right]+$ \\
\hline
\end{tabular}

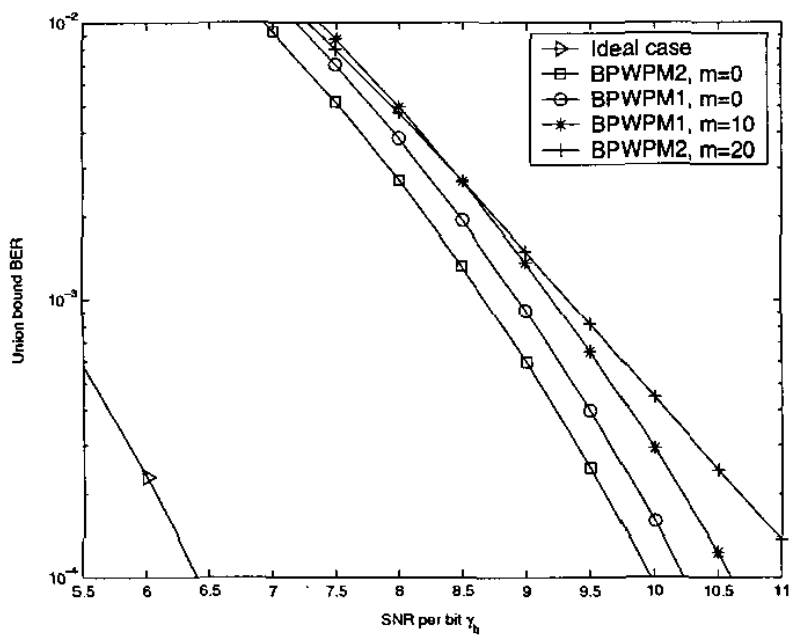

Fig. 2. Union bound BER of two signal sets inflicted with correlated jitter of $20 \mathrm{ps}$ RMS value and different $\mathrm{m}$.

is exponentially decaying in chip time index $n$. The order of Taylor approximation is set to 44 which guarantees the estimation error to be less than $0.001 \%$. The BER curves are shown in Fig. 2, we find that BPWPM2 signals have smaller degradation than BPWPM1 signals at low $\gamma_{b}$ when jitters are correlated. It results from the values of $R_{G_{6}}(\varepsilon)$ decay less than $R_{G_{7}}(\varepsilon)$ with small timing offset $\varepsilon$. Nevertheless, the advantage of BPWPM2 signals will disappear at high $\gamma_{b}$ due to the large equivalent additive variance in (17). When the jitter is independent $(m=0)$, the advantage of BPWPM2 signals will be more significant. The reason can be observed from (14). Since BPWPM2 have larger $N_{s}$ than BPWPM1, it should have smaller degradation. However, this advantage will be cancelled by larger $m$ if $m \neq 0$.

\section{CONCLUSION}

In this paper, we investigate the impact of correlated random timing jitters on impulse radio using 3 to $10 \mathrm{GHz}$ band. The analysis shows that the influence of jitter can be treated as another additive noise source, whose mean and variance can be efficiently approximated by Taylor series expansion and joint moments of Gaussian random variables. Two bipolar signal sets derived from the Gaussian pulses are used in this study. Both have the advantage of low receiver complexity. It is shown that not only the jitter RMS value but also the correlation between jitters influence the error rate performance. Inflicted with independent jitters of 20 ps RMS value, about $4 \mathrm{~dB}$ additional signal power must be added to achieve $10^{-4}$ BER. Even more power, which can be more than $1 \mathrm{~dB}$, must be added if the jitters are correlated. The effect of correlation is closely related to the pulse repetition time and the spreading factor. All these factors must be considered in impulse radio signal set design.

\section{REFERENCES}

[1] M. Hamalainen, V. Hovinen, R. Tesi, J. H. J. Iinatti, and M. Latva-aho, "On the UWB system coexistence with GSM900, UMTS/WCDMA, and GPS," IEEE J. Select. Areas Commun., vol. 20, no. 9, pp. 16921700 , Dec 2002.

12] "Revision of Part 15 the Commissions rules regarding ultra-wideband transmission systems," FCC ET Docket 98-153, 2002.

[3] W. M. Lovelace and J. K. Townsend, "The effects of timing jitter and tracking on the performance of impulse radio," IEEE J. Select. Areas Commun., vol. 20, no. 9, pp. 1646- 1651, Dec 2002

[4] A. Hajimiri, S. Limotyrakis, and T. H. Lee, "Jitter and phase noise in ring oscillators," IEEE J. Solid-State Circuits, vol. 34, no. 6, pp. $790-804$, Jun 1999.

[5] J. A. McNeill, "Jitter in ring oscillators," Ph.D. dissertation, Boston University, 1994.

[6] J. A. Bloom and T. R. Reed, "A gaussian derivative-based transform," IEEE Trans. Image Processing, vol. 5, no. 3, pp. 551 -553, March 1996.

[7] I. O'Donnell, S. W. Chen, B. T. Wang, and R. W. Brodersen, "An integrated, low power, ultra-wideband transceiver architecture for low-rate, indoor wireless systems," in IEEE CAS Workshop on Wireless Communications and Networking, Sep 2002.

[81 S. Budisin, "Golay complementary sequences are superior to PN sequences," in IEEE International Conference on Systems Engineering, Sep 1992, pp. $101-104$.

[9] J. M. Wozencraft and I. M. Jacobs, Principles of Communication Engineering. John Wiley, 1965.

[10] D. A. S. Fraser, Nomparametric methods in statistics. John Wiley, 1957.

111) R. L. Stratonovich, Topics in the theory of random noise, 2 nd ed. New York: Gordon and Breach, 1967. 